\title{
Estimation of neural voltage traces and associated variables in uncertain models
}

\author{
Pau Closas ${ }^{1 *}$, Antoni Guillamon ${ }^{2}$ \\ From Twenty Second Annual Computational Neuroscience Meeting: CNS*2013 \\ Paris, France. 13-18 July 2013
}

Measurements of membrane potential traces constitute the main observables to derive a biophysical neuron model; in particular, the dynamics of auxiliary variables and the model parameters are inferred from voltage traces, in a costly process that typically entails a variety of channel blocks and clamping techniques [1] and some uncertainty in the parameter values due to noise in the signal. Traces are also useful to obtain valuable information about synaptic input, an inverse problem with no satisfactory solution yet (see for instance [2,3]). In this presentation, we are interested in methods that can provide on-line estimation and avoid the need of
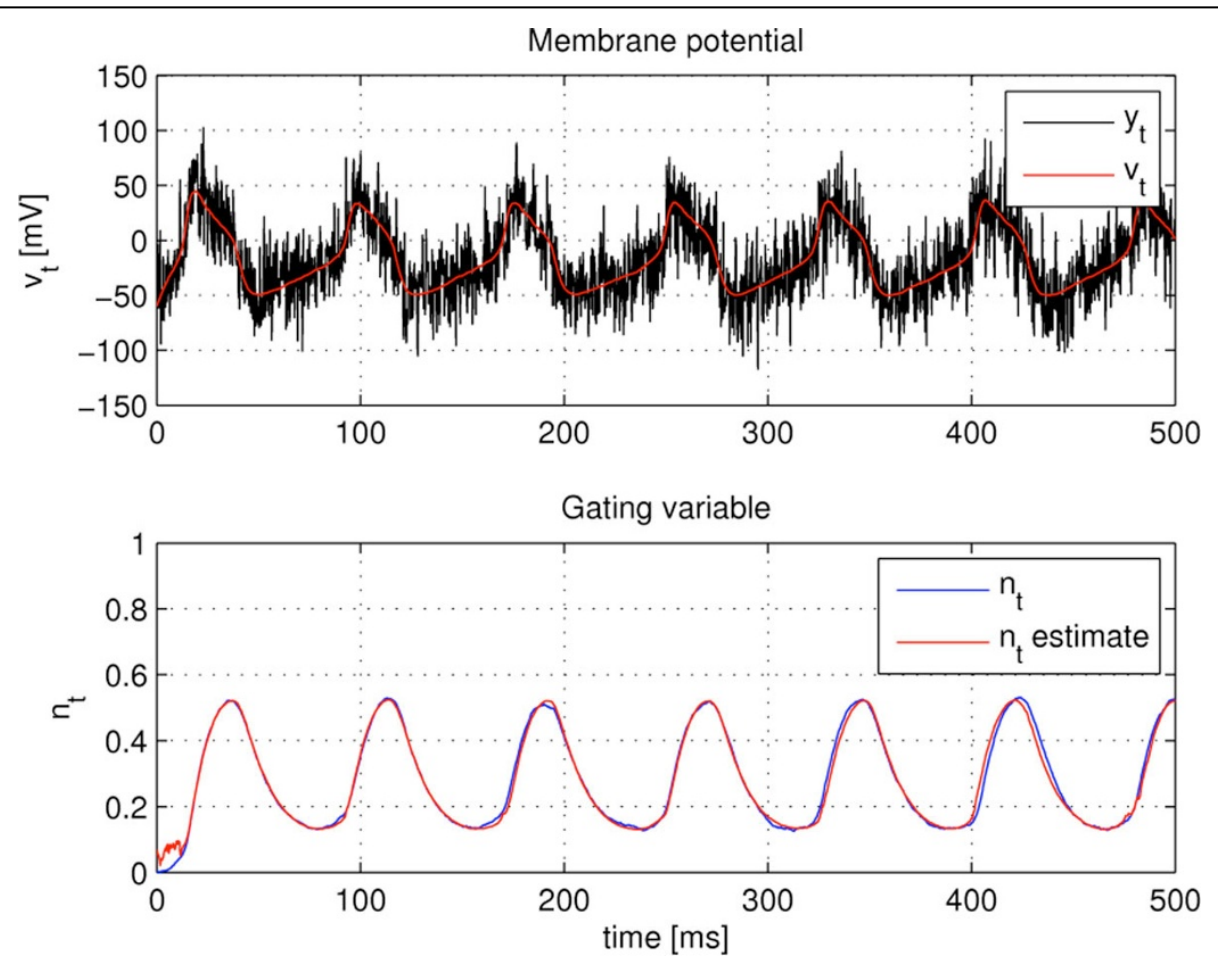

Figure 1 Top: Noisy membrane potential $\left(\mathbf{y}_{\mathrm{t}}\right)$ and estimated time-course $\left(\mathbf{v}_{\mathbf{t}}\right)$ with $\mathbf{5} \mathbf{d B}$ of signal-to-noise ratio. Bottom: time evolution of the true and the estimated $\mathrm{K}^{+}$gating variable $\left(\mathrm{n}_{\mathrm{t}}\right)$ in an uncertain Morris-Lecar model.

* Correspondence: pau.closas@cttc.cat

${ }^{1}$ Communications Subsystems Area, Centre Tecnològic de

Telecomunicacions de Catalunya (CTTC), Av. Carl Friedrich Gauss 7, 08860

Castelldefels, Barcelona, Catalonia, Spain

Full list of author information is available at the end of the article

○ 2013 Closas and Guillamon; licensee BioMed Central Ltd. This is an Open Access article distributed under the terms of the Creative 
repetitions that could be contaminated by neuronal variability. Particularly, we concentrate on methods to extract intrinsic activity of ionic channels, namely the probabilities of opening and closing ionic channels. We built a method based on Bayesian theory to sequentially infer these quantities from single-trace, noisy membrane potentials. A dynamical system is assumed for the evolution of the membrane potential and intrinsic variables of the model. Without loss of generality, we considered a Morris-Lecar neuron model to generate simulated data. The model is the a priori information of the Bayesian method, which is efficiently implemented by a particle filter (PF). The method is able to deal with the nonlinear nature of the problem and we observed its robustness to model inaccuracies. The proposed estimation method highly relies on the fact that the neuron model is known. This is true to some extent, but most of the parameters in the model are to be estimated beforehand (this holds for any model). Particularly, we assumed that the applied current, the maximal leakage conductance, and other parameters of the dynamical system were inaccurately known (error of 10\%) and model this uncertainty as an increase in the covariance matrix of the model. With the proposed PF with optimal importance density, we recuperated both the membrane potential and the activity of the potassium channel with the minimum attainable error. We derived the theoretical lower bound on the accuracy of any estimator and observed that it is achieved with the presented methodology, therefore the estimator is efficient. In conclusion, we propose a PF that is able to sequentially infer the timecourse of the membrane potential and the intrinsic activity of ionic channels from noisy observations of a voltage trace. The results show the validity of the approach and its statistical efficiency (see Figure 1). The procedure can be applied to any neuron model. Forthcoming applications are: combining the presented algorithm with fittings of voltage traces to neuron models; adding synaptic terms to the neuron model and use our method to infer the synaptic conductances. The latter is a challenging topic in the neuroscience literature, where we believe our PF method would give useful results to physiologists that aim at inferring brain's activation rules from neurons' activities.

\section{Acknowledgements}

P.C. has been partially supported by the Spanish Ministry of Economy and Competitiveness project TEC2012-39143 (SOSRAD), by the European Commission in the Network of Excellence in Wireless COMmunications NEWCOM\# (contract n. 318306). A.G. is supported by the MICINN/FEDER grant MTM2009-06973 (DACOBIAN) and the Generalitat de Catalunya CURDIUE grant number 2009SGR-859.

\section{Author details}

${ }^{1}$ Communications Subsystems Area, Centre Tecnològic de Telecomunicacions de Catalunya (CTTC), Av. Carl Friedrich Gauss 7, 08860 Castelldefels, Barcelona, Catalonia, Spain. ${ }^{2}$ Departament de Matemàtica Aplicada I, Universitat Politècnica de Catalunya (UPC), Carrer Doctor Marañón 44-50, 08028 Barcelona, Catalonia, Spain.
Published: 8 July 2013

\section{References}

1. Brette R, Destexhe A: Handbook of Neural Activity Measurement, Cambridge University Press 2012.

2. Rudolph M, Piwkowska Z, Badoual M, Bal T, Destexhe A: A method to estimate synaptic conductances from membrane potential fluctuations. J Neurophysiol 2004, 91:2884-2896.

3. Bédard C, Béhuret S, Deleuze C, Bal T, Destexhe A: "Oversampling method to extract excitatory and inhibitory conductances from single-trial membrane potential recordings,". J of neuroscience methods 2011.

doi:10.1186/1471-2202-14-S1-P151

Cite this article as: Closas and Guillamon: Estimation of neural voltage traces and associated variables in uncertain models. BMC Neuroscience 2013 14(Suppl 1):P151.

\section{Submit your next manuscript to BioMed Central and take full advantage of:}

- Convenient online submission

- Thorough peer review

- No space constraints or color figure charges

- Immediate publication on acceptance

- Inclusion in PubMed, CAS, Scopus and Google Scholar

- Research which is freely available for redistribution

Submit your manuscript at www.biomedcentral.com/submit
C Biomed Central 